\title{
1 Brain-Restricted mTOR Inhibition with Binary Pharmacology
}

3 Ziyang Zhang ${ }^{1}$, Qiwen Fan ${ }^{2,3}$, Xujun Luo ${ }^{2,3}$, Kevin J. Lou ${ }^{1}$, William A. Weiss ${ }^{2,3,4,5}$, Kevan

4 M. Shokat ${ }^{1, *}$

5

61 Department of Cellular and Molecular Pharmacology and Howard Hughes Medical

7 Institute, University of California, San Francisco, California

82 Helen Diller Family Comprehensive Cancer Center, San Francisco, California

$93^{3}$ Department of Neurology, University of California, San Francisco, California

104 Department of Pediatrics, University of California, San Francisco, California

115 Department of Neurological Surgery, University of California, San Francisco, California

12 *Corresponding author. kevan.shokat@ucsf.edu (K.M.S.). 


\section{Abstract}

14 On-target-off-tissue drug engagement is an important source of adverse effects

15 that constrains the therapeutic window of drug candidates. In diseases of the central

16 nervous system, drugs with brain-restricted pharmacology are highly desirable. Here we

17 report a strategy to achieve inhibition of mTOR while sparing mTOR activity elsewhere

18 through the use of a brain-permeable mTOR inhibitor RapaLink-1 and brain-impermeable

19 FKBP12 ligand RapaBlock. We show that this drug combination mitigates the systemic

20 effects of mTOR inhibitors but retains the efficacy of RapaLink-1 in glioblastoma

21 xenografts. We further present a general method to design cell-permeable, FKBP12-

22 dependent kinase inhibitors from known drug scaffolds. These inhibitors are sensitive to

23 deactivation by RapaBlock enabling the brain-restricted inhibition of their respective

24 kinase targets. 
Administration of a small molecule drug often leads to systemic pharmacological effects that contribute to both efficacy and toxicity and define its therapeutic index. While off-target effects may be mitigated by chemical modifications that improve the specificity of the drug, on-target-off-tissue toxicity represents a unique challenge that requires precise control over tissue partitioning. On-target-off-tissue toxicities affect commonly used drugs such as statins (myopathy caused by HMG CoA reductase inhibition in skeletal muscle) ${ }^{1}$ and first-generation antihistamines (drowsiness caused by $\mathrm{H}_{1}$ receptor blockade in the brain $)^{2}$ and can sometimes preclude the safe usage of otherwise effective

33 therapeutic agents. Chemical inhibitors of the mechanistic target of rapamycin (mTOR)

34 provide a case in point. Although mTOR inhibitors have shown efficacy in treating a

35 number of central nervous system (CNS) diseases including tuberous sclerosis complex 36 (TSC) $)^{3,4}$, glioblastoma $(\mathrm{GBM})^{5,6}$, and alcohol use disorder (AUD) $)^{7-9}$, systemic mTOR 37 inhibition is associated with a variety of dose-limiting adverse effects: immune 38 suppression, metabolic disorders, and growth inhibition in children ${ }^{10,11}$. If the 39 pharmacological effects of these mTOR inhibitors could be confined to the CNS, their 40 therapeutic window could be substantially widened. Here we present a chemical strategy

41 that allows brain-specific mTOR inhibition through the combination of two 42 pharmacological agents - a brain-permeable mTOR inhibitor (RapaLink-1) whose 43 function requires the intracellular protein FK506-binding protein 12 (FKBP12) and a brain-

44 impermeant ligand of FKBP12 (RapaBlock). When used in a glioblastoma xenograft 45 model, this drug combination drove tumor regression without detectable systemic toxicity.

46 We further demonstrate that this strategy can be adapted to achieve brain-specific 47 inhibition of other kinase targets by developing a method to convert known kinase 48 inhibitors into FKBP12-dependent formats.

Therapeutic targeting of mTOR kinase can be achieved both allosterically and 50 orthosterically. First-generation mTOR inhibitors rapamycin and its analogs (rapalogs) 51 bind to the FK506 rapamycin binding (FRB) domain of mTOR as a complex with the 52 intracellular protein FKBP12, resulting in substrate-dependent allosteric inhibition of 53 mTOR complex 12-14. Second-generation mTOR kinase inhibitors (TORKi) directly bind 54 in the ATP pocket of mTOR and inhibit the activity of both mTOR complex 1 and complex $552^{15-17}$. The third generation mTOR inhibitor RapaLink-1 is a bitopic ligand that 
56 simultaneously engages the ATP pocket and an allosteric pocket (the FRB domain) of

57 mTOR, achieving potent and durable inhibition of its kinase activity ${ }^{18}$. Despite its large

58 molecular weight (1784 Da), RapaLink-1 is cell- and brain-permeant and has shown

59 enhanced in vivo efficacy in driving glioblastoma regression compared to earlier mTOR

60 inhibitors $^{6}$.

61 Because RapaLink-1 contains a TOR kinase inhibitor (TORKi) moiety (Fig. 1a, grey

62 shades) that may directly bind in the ATP pocket, we wondered whether the interaction

63 of RapaLink-1 with FKBP12 is essential for its inhibition of mTOR. We performed in vitro

64 kinase assay with purified mTOR protein and found that RapaLink-1 exhibited identical

65 IC50 values as MLN0128 (the TORKi portion of RapaLink-1) and that inclusion of $10 \mu \mathrm{M}$

66 FKBP12 had no effect on its activity (Fig. 1b). Surprisingly, when we tested RapaLink-1

67 in cells, we observed a strong dependency on FKBP12 for mTOR inhibition. In

68 (K562/dCas9-KRAB cells), CRISPRi-mediated knockdown of FKBP12 with two distinct

69 single guide RNAs (sgRNAs) drastically impeded its cellular activity (Fig. 1c). This effect

70 was even more pronounced when we used FK506 (a high affinity natural ligand of

71 FKBP12) to pharmacologically block the ligand binding site of FKBP12. Similar FKBP12-

72 dependence of RapaLink-1 was observed when we monitored mTOR signaling by

73 Western Blot (Fig. 1d, see also ref $^{18}$ ) - whereas $10 \mathrm{nM}$ RapaLink-1 reduced P-S6

74 (S240/244) and P-4EBP(T37/46) to almost undetectable levels, combination of $10 \mathrm{nM}$

75 RapaLink-1 and $10 \mu$ M FK506 showed no effect on either marker.

76 These results highlight that while FKBP12 is not essential for RapaLink-1 to bind

77 and inhibit the active site of mTOR in vitro, it is required for cellular activity perhaps

78 serving as an intracellular sink for RapaLink-1 to accumulate in the cell. We probed this

79 possibility using a structural analog of RapaLink-1, where the TORKi moiety had been

80 replaced with tetramethylrhodamine (RapaTAMRA, Extended Data Fig. 1). This

81 fluorescent analog of RapaLink-1 allowed us to quantify intracellular compound

82 concentration by flow cytometry. Consistent with previous findings with RapaLink- ${ }^{18}$ and

83 other FKBP-binding compounds ${ }^{19,20}$, RapaTAMRA showed high cellular retention (10-fold

84 increase in median fluorescence intensity) even after extensive washout, but this effect

85 was diminished by FKBP12 knockdown (Extended Data Fig. 1c). While other factors may 
86 contribute, we believe that FKBP12-mediated cellular partitioning is partly responsible for

87 the exceptional potency of RapaLink-1.

We next considered whether the dependence of RapaLink-1 on FKBP12 could be harnessed to achieve CNS-restricted mTOR inhibition. Specifically, selective blockade

90 of FKBP12 in peripheral tissues with a potent, brain-impermeant small molecule ligand 91 would allow RapaLink to accumulate in the brain but not peripheral tissues, resulting in 92 brain-specific inhibition (Fig. 2a). To identify a candidate FKBP12 ligand with the suitable 93 permeability profile (i.e. cell-permeable but BBB-impermeable), we considered known 94 natural and synthetic high-affinity FKBP12 ligands (e.g., FK506²1,22, rapamycin ${ }^{23,24}$, 95 SLF/Shield-125,26), but all these compounds readily cross the blood-brain barrier (BBB). 96 Therefore, we synthesized a panel of derivatives of SLF and FK506 where polar 97 substituents (particularly hydrogen bond donors and acceptors) had been attached to the 98 solvent exposed parts of these two molecules, a design principle directly against the 99 empirical rules for designing BBB-permeable drugs ${ }^{27-30}$ (Extended Data Fig. 2). In 100 addition, modification of the C21-allyl of FK506 has the added advantage that it abolishes 101 the binding to calcineurin ${ }^{31}$, the natural target of FK506, whose inhibition causes 102 suppression of Nuclear factor of activated T-cells (NFAT) signaling and hence 103 immunosuppression (Fig. 2b). The majority of Shield-1 and FK506 derivatives we 104 synthesized maintained potent FKBP12 binding (Extended Data Fig . 2), as measured by 105 a competition fluorescence polarization assay ${ }^{32}$. We then screened these compounds $106(10 \mu \mathrm{M})$ in a cell-based assay, evaluating whether they can protect mTOR from inhibition 107 by RapaLink-1 (10 nM) by monitoring phospho-S6 level by Western Blot. While none of 108 the SLF derivatives were found to be effective, most FK506 analogs attenuated the 109 potency of RapaLink-1 (rescuing phospho-S6 levels), with some of them completely 110 blocking RapaLink-1 from inhibiting mTOR (Extended Data Fig. 2). Four of the most 111 effective RapaLink-1 blocking compounds were subjected to an additional in vivo screen, 112 where mTOR signaling was separately analyzed in the brain and skeletal muscle tissues 113 of mice treated with a combination of RapaLink-1 and the candidate compound (1 mg/kg 114 and $40 \mathrm{mg} / \mathrm{kg}$, respectively). A pyridine $\mathrm{N}$-oxide derivative of FK506 protected peripheral 115 tissues from RapaLink-1 but allowed potent inhibition of mTOR in the brain (Extended 
116 Data Fig. 3). We therefore chose this compound for our further study and refer to it as 117 "RapaBlock".

118 RapaBlock and FK506 bind to FKBP12 with comparable affinity (Fig. 2d, $K_{i}^{\prime}$ : 3.1

$119 \mathrm{nM}$ and $1.7 \mathrm{nM}$, respectively), but unlike FK506, RapaBlock does not exhibit any inhibitory 120 activity for calcineurin (Fig. 2e). In cultured cells, RapaBlock does not affect mTOR 121 signaling by itself (up to $10 \mu \mathrm{M}$ ) but attenuates the pharmacological effects of RapaLink1221 in a dose-dependent fashion (Fig. 3a). Interestingly, RapaBlock appears more effective 123 at blocking rapamycin, restoring phospho-S6 signal to the same level as untreated cells

124 at 100:1 stoichiometry (Fig. 3d). Because rapamycin and rapalogs exert 125 immunosuppressive effects by inhibiting mTOR activity and thus suppressing cell 126 proliferation in response to costimulatory and cytokine-mediated signals ${ }^{33,34}$, we asked 127 whether RapaBlock can prevent RapaLink-1-mediated immunosuppression. We 128 stimulated human peripheral mononuclear blood cells (PBMC) with anti-CD3 and anti129 CD28 antibodies in the presence of different concentrations of RapaLink-1 and 130 RapaBlock and assessed cell proliferation after 5 days. Whereas RapaLink-1 potently 131 inhibited PBMC proliferation at nanomolar concentrations, addition of RapaBlock 132 abolished this effect, shifting the $\mathrm{IC}_{50}$ by more than 100 -fold (Fig. 3b). Though mTOR 133 inhibition does not directly control cytokine production, we observed higher cumulative IL1342 release in cells co-treated with RapaLink-1 and RapaBlock compared to cells treated 135 with RapaLink-1 alone, presumably as a result of greater cell proliferation (Fig. 3c). 136 Consistent with our observation of mTOR signaling, RapaBlock appeared more effective 137 at protecting PBMC from rapamycin-mediated proliferation inhibition (Figs. 3e, 3f). 138 Together, these data demonstrate that by competitively binding to intracellular FKBP12, 139 RapaBlock renders RapaLink-1 and rapamycin incapable of inhibiting mTOR, and in so 140 doing diminishes the immunosuppressive effects of these two drugs.

141 To examine whether the combination of RapaLink-1 and RapaBlock allows brain142 specific inhibition of mTOR in vivo, we treated healthy BALB/c ${ }^{\text {nu/nu }}$ mice with RapaLink-1 143 (1 $\mathrm{mg} / \mathrm{kg})$ or a combination of RapaLink-1 (1 mg/kg) and RapaBlock (40 mg/kg), 144 stimulated mTOR activity with insulin $(250 \mathrm{mU})$ after $4 \mathrm{~h}$ or $24 \mathrm{~h}$, and analyzed dissected 145 tissues by immunoblot (Fig. 4a). RapaLink-1, when used as a single agent, potently 146 inhibited mTOR signaling in both skeletal muscle and brain tissues, as revealed by the 
147 reduced phosphorylation of S6 and 4EBP1. The combination of RapaLink-1 and

148 RapaBlock, however, exhibited remarkable tissue-specific effects: while mTOR activity in 149 the brain was inhibited at a comparable level to mice treated with RapaLink-1 only, mTOR 150 activity in skeletal muscle was not affected.

RapaLink-1 has been shown to be efficacious in orthotopic mouse models of 152 glioblastoma but inhibition of mTOR in the periphery does not contribute to efficacy. We 153 asked whether our combination regimen, lacking the ability to inhibit mTOR activity in 154 peripheral tissues, could retain the efficacy of RapaLink-1 in treating glioblastoma. We established orthotopic intracranial xenografts of U87MG cells expressing firefly luciferase in nude mice and treated these mice with i.p. injections of RapaLink-1 (1 mg/kg), 157 RapaBlock (40 mg/kg), or a combination of both every 5 days. All treatments were well 158 tolerated, and no significant changes of body weight were observed (Fig. 4b). RapaLink159 1, both as a single-agent or in combination with RapaBlock, significantly suppressed 160 tumor growth and improved survival, whereas RapaBlock alone had no significant effect 161 on either.

162 Having established a binary therapeutic approach to achieve brain-specific 163 inhibition of mTOR, we wondered whether the same strategy could be adapted to other 164 drugs for which brain-restricted pharmacology would be desirable. One critical challenge 165 is that for this approach to be generalizable, the "active" component (e.g. RapaLink-1) 166 must be dependent on the availability FKBP12. Drugs with this property are rare, few if 167 any besides rapamycin and FK506 are known. Our earlier investigation of RapaLink-1 168 (Fig. 1) and RapaTAMRA (Extended Data Fig. 1) led us to hypothesize that other 169 bifunctional molecules consisting of a FKBP12-binding moiety and a kinase inhibitor 170 moiety could be conditionally active and amenable to modulation with RapaBlock.

171 We first tested our hypothesis with GNE7915, a potent and specific inhibitor of 172 leucine-rich repeat kinase 2 (LRRK2) a pre-clinical agent under investigation for the 173 treatment of Parkinson's disease ${ }^{35-37}$. As gain-of-function mutations of LRRK2 are 174 strongly associated with hereditary and sporadic forms of Parkinson's diseases, LRRK2 175 kinase inhibition has been pursued as a potential therapeutic strategy ${ }^{38,39}$. However, 176 recent studies demonstrating that systemic LRRK2 inhibition with small molecule 177 inhibitors induced cytoplasmic vacuolation of type II pneumocytes suggests a potential 
178 safety liability for these compounds ${ }^{40,41}$. We synthesized FK-GNE7915 by chemically 179 linking the pharmacophores of FK506 and GNE7915 with a piperazine group (Fig. 5a). 180 In in vitro LRRK2 kinase assays, FK-GNE7915 was an inferior inhibitor to GNE7915 (Fig. $1815 \mathrm{c}$, IC50s of $107 \mathrm{nM}$ and $3.0 \mathrm{nM}$, respectively), though its activity was potentiated by 182 including $10 \mu \mathrm{M}$ FKBP12 in the assay $\left(\mathrm{IC}_{50}: 21 \mathrm{nM}\right)$. However, in a cellular assay where 183 we quantified phospho-LRRK2 (S935) levels as a marker for LRRK2 inhibition, FK184 GNE7915 was more potent than the parent compound GNE7915 by more than 10 -fold 185 (Fig $5 \mathrm{~d}$, IC50s of $6.7 \mathrm{nM}$ and $81 \mathrm{nM}$, respectively). The juxtaposition of these two results 186 suggested a role of the FK506 moiety in the enhanced cellular potency of FK-GNE7915. 187 We reasoned that the high-affinity FK506-FKBP12 interaction can promote the 188 intracellular accumulation of FK-GNE7915 similar to the case of RapaLink-1 and 189 confirmed this using a bifunctional fluorescent probe FK-TAMRA (Extended Data Fig. 4). 190 This feature allowed us to program LRRK2 inhibition by controlling the availability of 191 FKBP12: whereas 100 nM FK-GNE7915 treatment reduced phosphor-LRRK2 (S935) 192 level to $15 \%$ of basal level, the combination of $100 \mathrm{nM}$ FK-GNE7915 and $1 \mu \mathrm{M}$ RapaBlock 193 had no effect on LRRK2 activity (Fig. 5e).

194 The successful conversion of GNE7915 into a FKBP-dependent LRRK2 inhibitor 195 by simply linking it to an FK506 fragment prompted us to evaluate the generality of this 196 strategy. We explored a number of kinase inhibitors that are being investigated for CNS197 diseases: dasatinib (Src family kinase inhibitor, glioblastoma) ${ }^{42-44}$, lapatinib (EGFR/HER2 198 inhibitor, glioblastoma) ${ }^{45,46}$, and prostetin (MAP4K4 inhibitor, amyotrophic lateral sclerosis 199 and Alzheimer's disease $)^{47}$. In all three cases, chemically linking the kinase inhibitor to 200 FK506 yielded bifunctional molecules that retained the kinase inhibitory activities of the 201 parent molecules (Extended Data Figs. 5-7). For FK-dasatinib, we also examined its 202 target specificity using both biochemical (Invitrogen SelectScreen Kinase Profiling) and 203 live-cell kinase profiling ${ }^{48}$ and observed an identical spectrum of kinase targets as 204 dasatinib with the exception of DDR2, a known off-target of dasatinib (Extended Data Fig. 205 5). Despite their large molecular weights, these bifunctional molecules are active in cells 206 with comparable potencies to their parent compounds and sensitive to deactivation by 207 RapaBlock. While these examples represent a limited set of kinase inhibitors with 
208 potential CNS disease indications, it is conceivable more drugs can be similarly 209 configured with programmable pharmacology without losing cellular potency.

\section{Conclusion}

212 Exploiting the unique functional dependence of rapamycin analogs on FKBP12, we

213 have developed an approach to achieve brain-specific mTOR inhibition through the 214 simultaneous administration of a potent mTOR inhibitor (RapaLink-1) and a cell215 permeable, BBB-impermeable ligand of FKBP12 (RapaBlock). Tissue-restricted mTOR 216 inhibition enabled by this binary pharmacology strategy reduces the toxicity in peripheral 217 tissues but maintains therapeutic benefits of RapaLink-1 in glioblastoma xenograft 218 models. On the basis of these findings, it seems reasonable to anticipate that the same 219 drug combination may be of broader value in other CNS diseases driven by dysregulated 220 mTOR activity such as Alcohol Use Disorder.

221 The applicability of our approach extends beyond mTOR inhibition. We show that chemically linking ATP-site kinase inhibitors to FK506 through solvent-exposed groups

223 leads to a new class of cell-permeable kinase inhibitors whose activity depends on the 224 abundant endogenous protein FKBP12. These inhibitors are characterized by their ability 225 to mediate the formation of a ternary complex of the drug, the target kinase and FKBP12, 226 as well as their amenability to activity modulation by RapaBlock. Further in vivo studies 227 and medicinal chemistry are necessary to assess and optimize these compounds on a 228 case-by-case basis, but our initial investigations show that it is feasible to attain brain229 selective kinase inhibition of LRRK2 using a FKBP-dependent kinase inhibitor (FK230 GNE7915) and RapaBlock.

231 We have investigated the mechanism by which RapaBlock controls the cellular 232 activity of RapaLink-1 as well as other FKBP-dependent kinase inhibitors. Our current 233 data has revealed at least two roles of cellular FKBP12 in the function of RapaLink-1 (and 234 other FKBP-binding compounds). First, we have shown that FKBP12 serves as a 235 reservoir to retain and accumulate RapaLink-1 inside the cell, achieving exceptional 236 cellular concentration. Second, for most FKBP-dependent kinase inhibitors we have 237 investigated, FKBP12 improves their potency in cell-free assays. Though we have not 238 obtained direct evidence, we hypothesize that in aqueous solutions, a bifunctional 
compound built with flexible linkers between hydrophobic pharmacophores will mainly adopt a binding-incompetent conformation to minimize hydration penalty ("hydrophobic 241 collapse"), and binding of FKBP12 will expose the inhibitor moiety to enable target 242 inhibition. RapaBlock impedes both of these processes by occupying the ligand-binding 243 site of FKBP12. While further investigation is clearly necessary to elucidate how these 244 high molecular weight compounds enter cells and whether FKBP12 participates in the 245 binding interaction with the target protein, we believe this system provides a generalizable 246 approach for programmable kinase inhibition.

247 Combining two pharmaceutical agents to achieve tissue-selective therapeutic 248 effects has been previously employed in drugs approved for clinical use or in development.

249 Examples include Levodopa/Carbidopa (BBB-permeable dopamine precursor/BBB250 impermeable dopa decarboxylase inhibitor) for Parkinson's Disease ${ }^{49}$, conjugated 251 estrogens/bazedoxifene (BBB-permeable estrogen/BBB-impermeable estrogen receptor 252 modulator) for post-menopausal hot flashes and osteoporosis ${ }^{50}$, and 253 donepezil/solifenacin (BBB-permeable acetylcholinesterase inhibitor/BBB-impermeable 254 anticholinergic) for Alzheimer's disease ${ }^{51}$. Our approach differs from these precedents in 255 that it does not involve two drugs with counteracting effects on the same target or pathway; 256 instead, RapaBlock controls tissue-specificity by directly attenuating the activity of the 257 kinase inhibitor. The present system therefore has the advantage of being adaptable for 258 a multitude of targets, only requiring an invariant RapaBlock molecule and a FKBP12259 dependent inhibitor that can be readily designed based on the structures of FK506 and 260 lead compounds. While we have focused on protein kinases in this study, it seems 261 reasonable to expect that the approach is also applicable to other classes of therapeutic 262 targets, such as GTPases and histone modification enzymes.

\section{Acknowledgements}

265 We thank Douglas Wassarman for discussions. Z.Z. is a Damon Runyon Fellow 266 supported by the Damon Runyon Cancer Research Foundation (DRG-2281-17).

267 K.M.S, Q.W.F. and W.A.W. acknowledge NIH 1R01CA221969. K.M.S, and W.A.W. 268 acknowledge the Samuel Waxman Cancer Research Foundation. W.A.W. acknowledges 
270 J. Fox Foundation P0536220, The Mark Foundation for Cancer Research and the Howard

271 Hughes Medical Institute.

272

\section{Author Contributions}

274 Z.Z., Q.F., W.A.W and K.M.S. conceived the project, designed and analyzed the 275 experiments, and wrote the manuscript. Z.Z., Q.F., X.L. and K.J.L. performed the 276 laboratory experiments. W.A.W. supervised the in vivo experiments.

278 Supplementary Information is available for this paper.

\section{Author Information}

281 Correspondence and requests for materials should be addressed to K.M.S.

282 (kevan.shokat@ucsf.edu). 


\section{References}

1. Johnson, T. E. et al. Statins induce apoptosis in rat and human myotube cultures by inhibiting protein geranylgeranylation but not ubiquinone. Toxicol. Appl. Pharmacol. 200, 237-250 (2004).

2. Reiner, P. B. \& Kamondi, A. Mechanisms of antihistamine-induced sedation in the human brain: $\mathrm{H} 1$ receptor activation reduces a background leakage potassium current. Neuroscience 59, 579-588 (1994).

3. $\mathrm{Li}, \mathrm{M}$. et al. Efficacy and safety of mTOR inhibitors (rapamycin and its analogues) for tuberous sclerosis complex: A meta-analysis. Orphanet J. Rare Dis. 14, 1-9 (2019).

4. Franz, D. N. et al. Rapamycin causes regression of astrocytomas in tuberous sclerosis complex. Ann. Neurol. 59, 490-498 (2006).

5. Akhavan, D., Cloughesy, T. F. \& Mischel, P. S. Learned From Bench To Bedside. Neuro. Oncol. 12, 882-889 (2010).

6. Fan, Q. W. et al. A Kinase Inhibitor Targeted to mTORC1 Drives Regression in Glioblastoma. Cancer Cell 31, 424-435 (2017).

7. Neasta, J., Hamida, S. Ben, Yowell, Q., Camicella, S. \& Ron, D. Role for mammalian target of rapamycin complex 1 signaling in neuroadaptations underlying alcohol-related disorders. Proc. Natl. Acad. Sci. U. S. A. 107, 2009320098 (2010).

8. Barak, S. et al. Disruption of alcohol-related memories by mTORC1 inhibition prevents relapse. Nat. Neurosci. 16, 1111-1117 (2013).

9. Morisot, N., Novotny, C. J., Shokat, K. M. \& Ron, D. A new generation of mTORC1 inhibitor attenuates alcohol intake and reward in mice. Addict. Biol. 23, 713-722 (2018).

10. Pallet, N. \& Legendre, C. Adverse events associated with mTOR inhibitors. Expert Opin. Drug Saf. 12, 177-186 (2013).

11. González, D. et al. Growth of kidney-transplanted pediatric patients treated with sirolimus. Pediatr Nephrol 26, 961-966 (2011).

12. Chiu, M. I., Katz, H. \& Berlin, V. RAPT1, a mammalian homolog of yeast Tor, interacts with the FKBP12/rapamycin complex. Proc. Natl. Acad. Sci. U. S. A. 91, 12574-12578 (1994).

13. Loewith, R. et al. Two TOR complexes, only one of which is rapamycin sensitive, have distinct roles in cell growth control. Mol. Cell 10, 457-468 (2002).

14. Gaubitz, C. et al. Molecular Basis of the Rapamycin Insensitivity of Target Of Rapamycin Complex 2. Mol. Cell 58, 977-988 (2015).

15. Feldman, M. E. et al. Active-site inhibitors of mTOR target rapamycin-resistant outputs of mTORC1 and mTORC2. PLoS Biol. 7, 0371-0383 (2009).

16. García-Martínez, J. M. et al. Ku-0063794 is a specific inhibitor of the mammalian target of rapamycin (mTOR). Biochem. J. 421, 29-42 (2009).

17. Thoreen, C. C. et al. An ATP-competitive mammalian target of rapamycin inhibitor reveals rapamycin-resistant functions of mTORC1. J. Biol. Chem. 284, 8023-8032 (2009).

18. Rodrik-Outmezguine, V. S. et al. Overcoming mTOR resistance mutations with a new-generation mTOR inhibitor. Nature 534, 272-6 (2016). 
19. Marinec, P. S. et al. FK506-binding protein (FKBP) partitions a modified HIV protease inhibitor into blood cells and prolongs its lifetime in vivo. Proc. Natl. Acad. Sci. U. S. A. 106, 1336-1341 (2009).

20. Orange, D. E. et al. Dendritic cells loaded with FK506 kill T cells in an antigenspecific manner and prevent autoimmunity in vivo. Elife 2, 1-13 (2013).

21. Liu, J. et al. Calcineurin is a common target of cyclophilin-cyclosporin A and FKBPFK506 complexes. Cell 66, 807-815 (1991).

22. Griffith, J. P. et al. X-ray structure of calcineurin inhibited by the immunophilinimmunosuppressant FKBP12-FK506 complex. Cell 82, 507-522 (1995).

23. Brown, E. J. et al. A mammalian protein targeted by G1-arresting rapamycinreceptor complex. Nature 369, 756-758 (1994).

24. Sabatini, D. M., Erdjument-Bromage, H., Lui, M., Tempst, P. \& Snyder, S. H. RAFT1: A mammalian protein that binds to FKBP12 in a rapamycin-dependent fashion and is homologous to yeast TORs. Cell 78, 35-43 (1994).

25. Holt, D. A. et al. Design, Synthesis, and Kinetic Evaluation of High-Affinity FKBP Ligands. J. Am. Chem. Soc. 115, 9925-9938 (1993).

26. Banaszynski, L. A., Chen, L. chun, Maynard-Smith, L. A., Ooi, A. G. L. \& Wandless, T. J. A Rapid, Reversible, and Tunable Method to Regulate Protein Function in Living Cells Using Synthetic Small Molecules. Cell 126, 995-1004 (2006).

27. Wager, T. T. et al. Defining desirable central nervous system drug space through the alignment of molecular properties, in vitro ADME, and safety attributes. ACS Chem. Neurosci. 1, 420-434 (2010).

28. Rankovic, Z. CNS Drug Design: Balancing Physicochemical Properties for Optimal Brain Exposure. J. Med. Chem. 58, 2584-2608 (2015).

29. Banks, W. A. Characteristics of compounds that cross the blood-brain barrier. BMC Neurol. 9, S3 (2009).

30. Pajouhesh, H. \& Lenz, G. R. Medicinal chemical properties of successful central nervous system drugs. NeuroRx 2, 541-553 (2005).

31. Clemons, P. A. et al. Synthesis of Calcineurin-Resistant Derivatives of FK506 and Selection of Compensatory Receptors. 9, 49-61 (2002).

32. Kozany, C., März, A., Kress, C. \& Hausch, F. Fluorescent probes to characterise FK506-binding proteins. ChemBioChem 10, 1402-1410 (2009).

33. Dumont, F. J., Staruch, M. J., Koprak, S. L., Melino, M. R. \& Sigal, N. H. Distinct mechanisms of suppression of murine $T$ cell activation by the related macrolides FK-506 and rapamycin. J. Immunol. 144, 251-8 (1990).

34. Powell, J. D. \& Delgoffe, G. M. The Mammalian Target of Rapamycin: Linking T Cell Differentiation, Function, and Metabolism. Immunity 33, 301-311 (2010).

35. Repeat, R. et al. Discovery of Highly Potent, Selective, and Brain-Penetrable Leucine- Rich Repeat Kinase 2 (LRRK2) Small Molecule Inhibitors. J. Medicial Chem. 2, 1-6 (2012).

36. Kavanagh, M. E., Doddareddy, M. R. \& Kassiou, M. The development of CNSactive LRRK2 inhibitors using property-directed optimisation. Bioorganic Med. Chem. Lett. 23, 3690-3696 (2013).

37. Estrada, A. A. et al. Discovery of highly potent, selective, and brain-penetrant aminopyrazole Leucine-rich repeat kinase 2 (LRRK2) small molecule inhibitors. J. Med. Chem. 57, 921-936 (2014). 
375

382

383

384

385

386

387

388

389

390

391

392

393

394

395

396

397

398

399

400

401

402

403

404

405

406

407

408

409
38. Lee, B. D., Dawson, V. L. \& Dawson, T. M. Leucine-rich repeat kinase 2 (LRRK2) as a potential therapeutic target in Parkinson's disease. Trends Pharmacol. Sci. 33, 365-373 (2012).

39. Rudenko, I. N., Chia, R. \& Cookson, M. R. Is inhibition of kinase activity the only therapeutic strategy for LRRK2-associated Parkinson's disease? BMC Med. 10, $1-$ 8 (2012).

40. Fuji, R. N. et al. Effect of selective LRRK2 kinase inhibition on nonhuman primate lung. Sci. Transl. Med. 7, 273ra15 (2015).

41. Baptista, M. A. S. et al. LRRK2 kinase inhibitors induce a reversible effect in the lungs of non-human primates with no measurable pulmonary deficits. bioRxiv 390815 (2018). doi:10.1101/390815

42. Lombardo, L. J. et al. Discovery of N-(2-chloro-6-methylphenyl)-2-(6-(4-(2hydroxyethyl)- $\quad$ piperazin-1-yl)-2-methylpyrimidin-4-ylamino)thiazole-5carboxamide (BMS-354825), a dual Src/Abl kinase inhibitor with potent antitumor activity in preclinical assays. J. Med. Chem. 47, 6658-6661 (2004).

43. Lassman, A. B. et al. Phase 2 trial of dasatinib in target-selected patients with recurrent glioblastoma (RTOG 0627). Neuro. Oncol. 17, 992-998 (2015).

44. Schiff, D. \& Sarkaria, J. Dasatinib in recurrent glioblastoma: Failure as a teacher. Neuro. Oncol. 17, 910-911 (2015).

45. Medina, P. J. \& Goodin, S. Lapatinib: A dual inhibitor of human epidermal growth factor receptor tyrosine kinases. Clin. Ther. 30, 1426-1447 (2008).

46. Thiessen, B. et al. A phase I/II trial of GW572016 (lapatinib) in recurrent glioblastoma multiforme: Clinical outcomes, pharmacokinetics and molecular correlation. Cancer Chemother. Pharmacol. 65, 353-361 (2010).

47. Bos, P. H. et al. Development of MAP4 Kinase Inhibitors as Motor NeuronProtecting Agents. Cell Chem. Biol. 26, 1703-1715.e37 (2019).

48. Zhao, Q. et al. Broad-spectrum kinase profiling in live cells with lysine-targeted sulfonyl fluoride probes. J. Am. Chem. Soc. 139, 680-685 (2017).

49. Bartholini, G., Burkard, W. P., Pletscher, A. \& Bates, H. M. Increase of cerebral catecholamines caused by 3,4-dihydroxyphenylalanine after inhibition of peripheral decarboxylase [14]. Nature 215, 852-853 (1967).

50. Komm, B. S. \& Mirkin, S. Evolution of the tissue selective estrogen complex (TSEC). J. Cell. Physiol. 228, 1423-1427 (2013).

51. Chase, T. N., Farlow, M. R. \& Clarence-Smith, K. Donepezil Plus Solifenacin (CPC201) Treatment for Alzheimer's Disease. Neurotherapeutics 14, 405-416 (2017). 
a

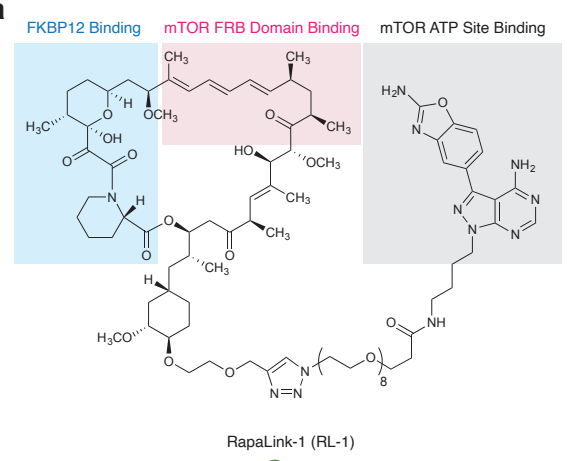

ar

d

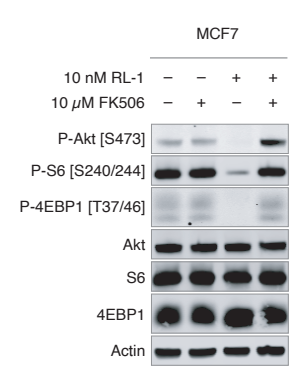

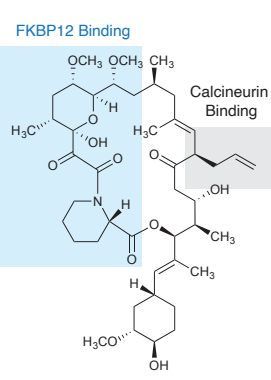

FK506

$(3$

e

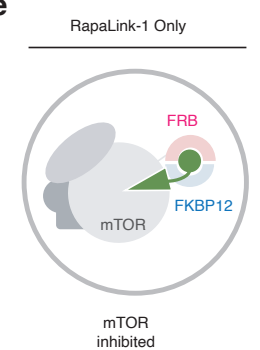

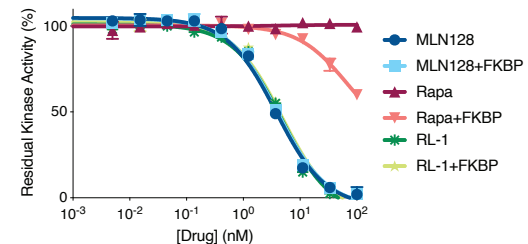
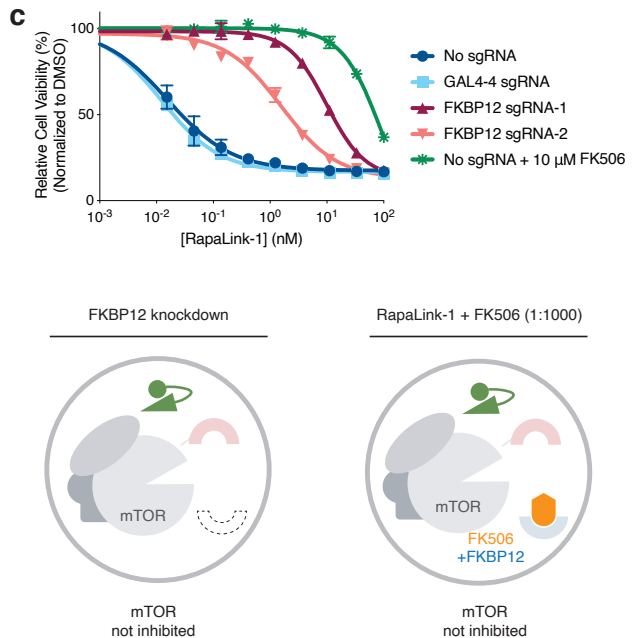

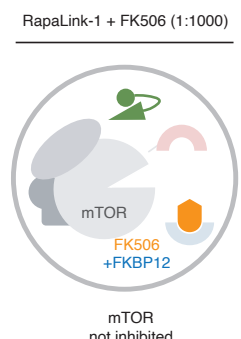

411 Figure 1. RapaLink-1 is a potent mTOR inhibitor that requires FKBP12 for its

412 cellular activity. a, Chemical structures of RapaLink-1 and FK506. b, Inhibition of mTOR 413 activity by MLN128, RapaLink-1 (RL-1) or Rapamycin in the presence or absence of 10 $414 \mu \mathrm{M}$ FKBP12 in vitro kinase assay. c, K562-dCas9-KRAB cells transduced with GAL4-4 415 (control) or FKBP12-targeting sgRNAs were treated with RapaLink-1 and cell proliferation 416 was assessed after $72 \mathrm{~h}$. In the last listed condition, cells were transduced with sgRNA 417 but treated with RapaLink-1 in the presence of $10 \mu \mathrm{M}$ FK506. d, Immunoblot analysis of 418 mTOR signaling in MCF7 cells treated with DMSO, RapaLink-1, FK506, or a combination 419 of RapaLink-1 and FK506. e, Schematics of the proposed working model. Grey circles 420 indicate cellular membranes. 
a

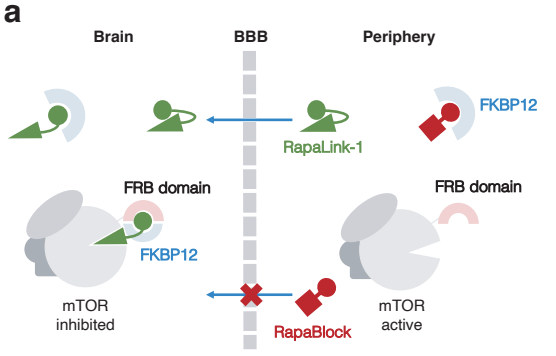

d

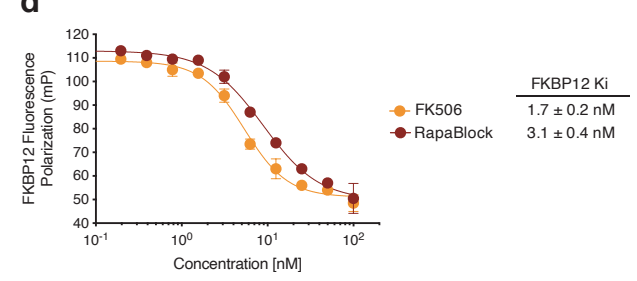

b

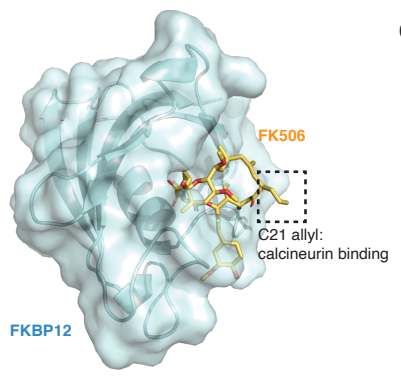

e c
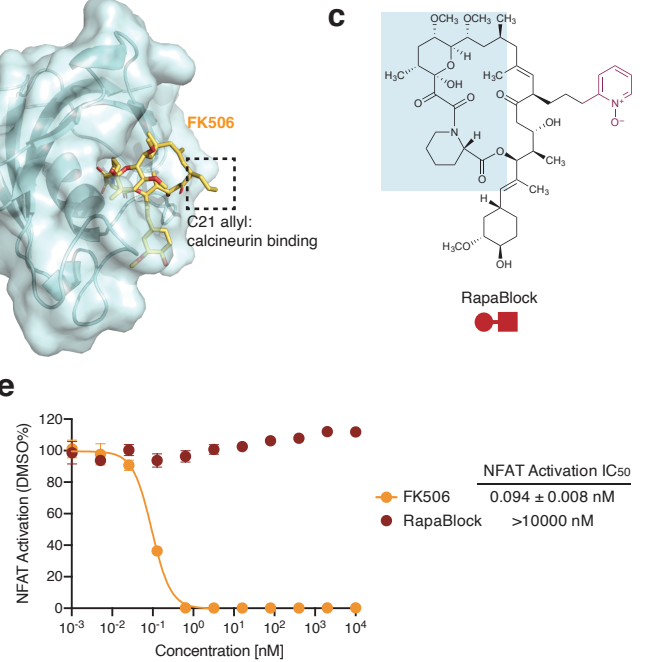

423 Figure 2. RapaBlock is a potent, non-immunosuppressive FKBP12 ligand. a, a 424 proposed model to achieve brain-specific mTOR inhibition through the combination of an 425 FKBP12-dependent mTOR inhibitor (RapaLink-1) and a brain impermeable FKBP12 426 ligand (RapaBlock). b, a FK506-FKBP12 co-crystal structure (PDB: 1FKJ) showing that 427 the C21 allyl group is solvent exposed and its modification may lead to abolished 428 calcineurin binding. c, chemical structures RapaBlock. Blue shaded areas indicate the 429 FKBP12-binding moiety. d, competition fluorescence polarization assay using 430 fluorescein-labeled rapamycin as the tracer compound $(n=3)$. e, Jurkat cells expressing 431 a luciferase under the control of NFAT transcription response element were stimulated 432 with phorbol myristate acetate and ionomycin in the presence of various concentrations 433 of compounds $(n=3)$. 
a

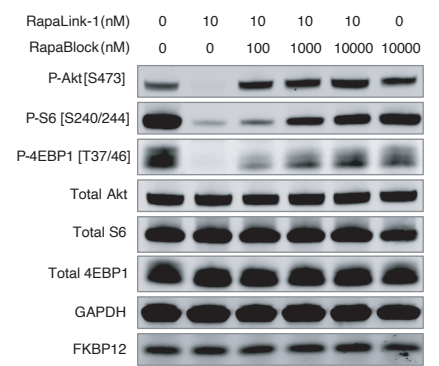

d

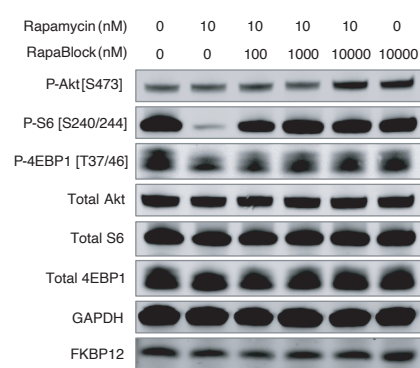

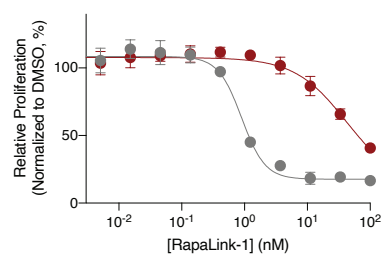

- No RapaBlock

e

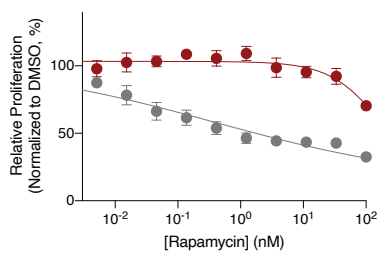

- No RapaBlock
C

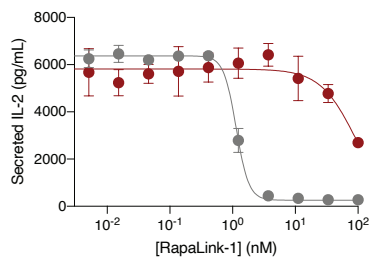

- +10 HM RapaBlock

f

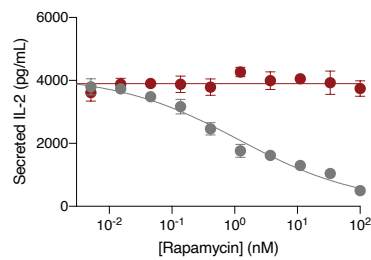

- $+10 \mu \mathrm{M}$ RapaBlock

435 Figure 3. RapaBlock protects cells from mTOR inhibition by RapaLink-1 and

436 Rapamycin. a, d, MCF7 cells were treated with a combination of RapaLink-1 and 437 RapaBlock (a), or Rapamycin and RapaBlock (d) for $4 \mathrm{~h}$, then phosphorylation of mTOR 438 substrates were analyzed by immunoblotting. Results shown are representative of three 439 independent experiments. b, e, Human PBMCs were stimulated with anti-CD3 and anti440 CD28 in the presence of varying amounts of RapaLink-1 and RapaBlock (b) or rapamycin 441 and RapaBlock (e), and cell proliferation was measured after 120 h. c, f, Interleukin-2 442 secretion in the culture supernatant of PBMCs in $\mathbf{c}$ and $\mathbf{f}$ was quantified by sandwich 443 ELISA. 
a

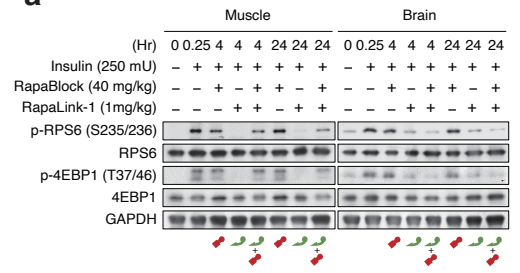

b

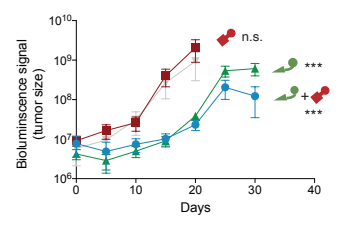

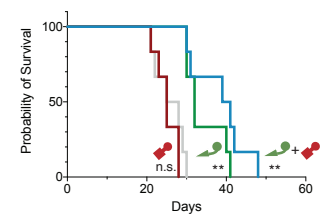

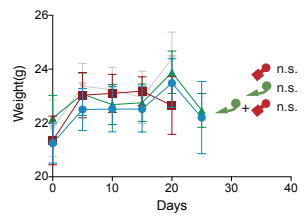

445 Figure 4. Cotreatment with RapaLink-1 and RapaBlock allows brain-specific

446 inhibition of mTOR. a, Mice were treated intraperitoneally with a single dose of 447 RapaLink-1 (1 mg/kg), RapaBlock (40 mg/kg), or a combination of both. mTOR activity 448 was stimulated with insulin $(250 \mathrm{mU}) 15$ minutes before tissue dissection. Whole brain 449 and skeletal muscle were analyzed by immunoblot ( $n=3$ except for the following: no450 insulin group, $n=1$; insulin-only group, $n=2$. Results are shown for one animal from each 451 group). b, Mice bearing luciferase-expressing orthotopic glioblastoma xenografts 452 (U87MG-Luc) were randomized to four different groups $(n=7)$ and treated 453 intraperitoneally every 5 days: (1) vehicle; (2) RapaBlock (40 mg/kg); (3) RapaLink-1 (1 $454 \mathrm{mg} / \mathrm{kg}$ ); (4) RapaLink-1 (1 mg/kg) and RapaBlock (40 mg/kg). Tumor size was monitored 455 every 5 days using bioluminescence imaging. Statistical tests were performed for each 456 treatment group versus vehicle-treated group: unpaired Student's $t$-test (tumor size on 457 day 20); log rank test (survival). n.s., not significant; ${ }^{* *}, p \leq 0.01 ;{ }^{* * *}, p \leq 0.001$. 
a

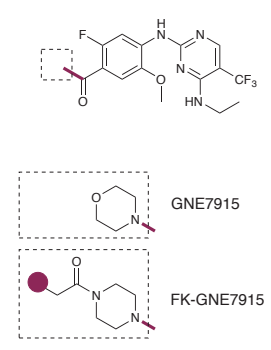

C

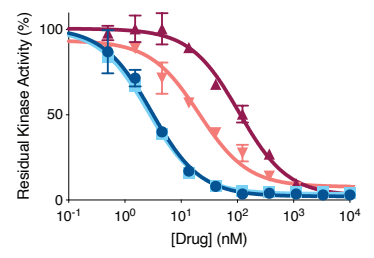

- GNE7915 GNE7915+10 $\mu \mathrm{M}$ FKBP12

—FK-GNE7915 + FK-GNE7915+ $10 \mu \mathrm{M}$ FKBP12

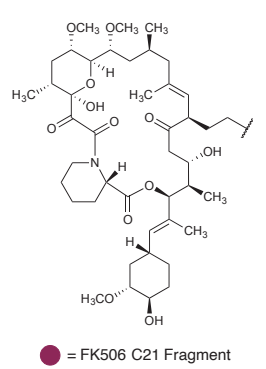

d

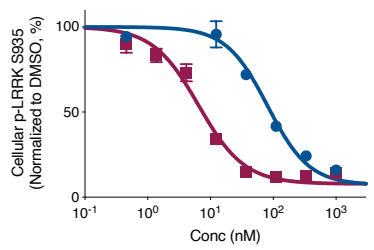

— GNE7915
— FK-GNE7915 b

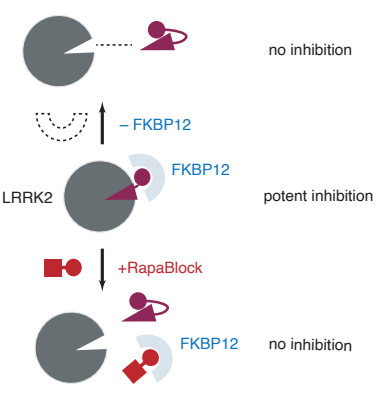

e

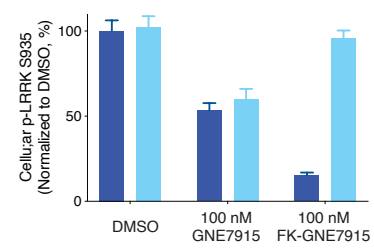

No RrapaBlock
$+1 \mu \mathrm{M}$ RapaBlock

459 Figure 5. Programmable kinase inhibition with FKBP-dependent kinase inhibitors 460 and RapaBlock. a, Structures of GNE7915 and FK-GNE7915. b, Proposed working 461 model for FKBP-dependent kinase inhibitors. c, kinase inhibition in the absence or 462 presence of supplemented $10 \mu \mathrm{M}$ recombinant FKBP12 protein. Data is the average of 463 two replicates. d, e, RAW264.7 cells were treated with GNE7915, FK-GNE7915, and/or 464 RapaBlock and phospho-LRRK2 (S935) was analyzed by time-resolved FRET using 465 epitopically orthogonal antibodies for LRRK2 and p-LRRK2 (S935). See Supplementary 466 Information for experimental details. 
a

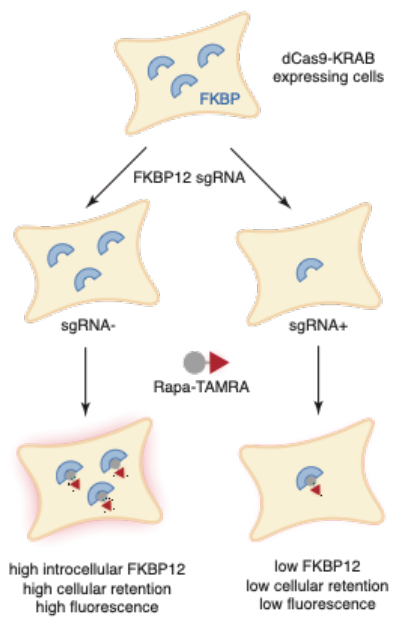

b

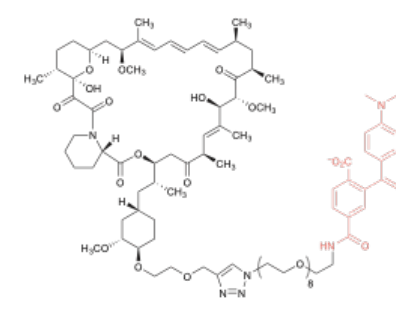

c

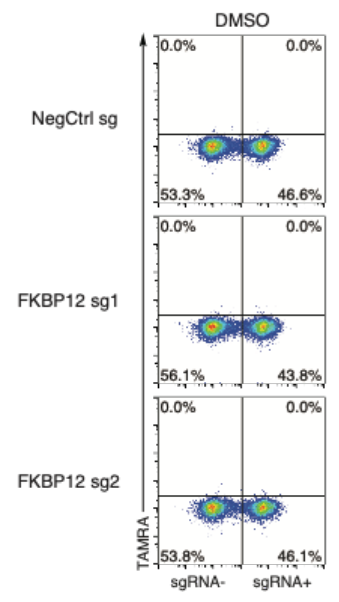

DMSO

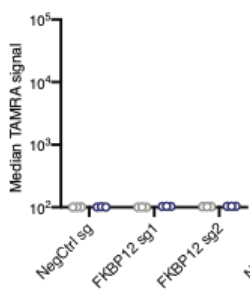

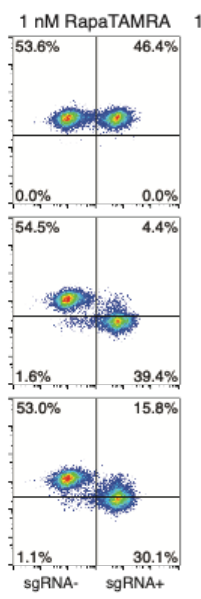

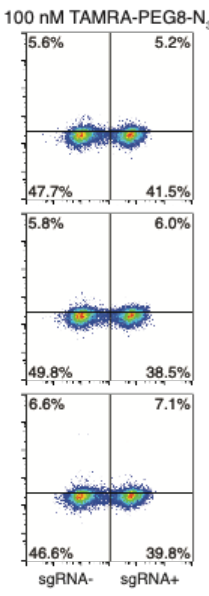

$1 \mathrm{nM}$ RapaTAMRA $100 \mathrm{nM}$ TAMRA-PEG8- $\mathrm{N}_{3}$

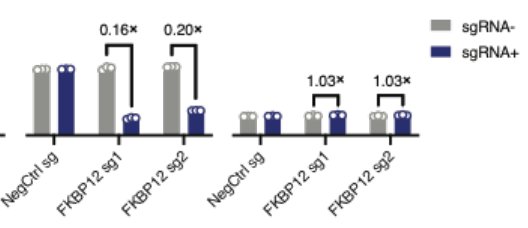

468 Extended Data Figure 1. FKBP12-Rapamycin interaction contributes to cellular 469 accumulation of a fluorescent analog of RapaLink-1. a, Illustration of the flow 470 cytometry-based assay to assess cellular accumulation of TAMRA compounds. b, 471 Structure of a fluorescent probe Rapa-TAMRA. c, FKBP12 knockdown decreases 472 cellular retention of Rapa-TAMRA, but not TAMRA-PEG8-N3. See Supplementary 473 Information for gating strategy. 


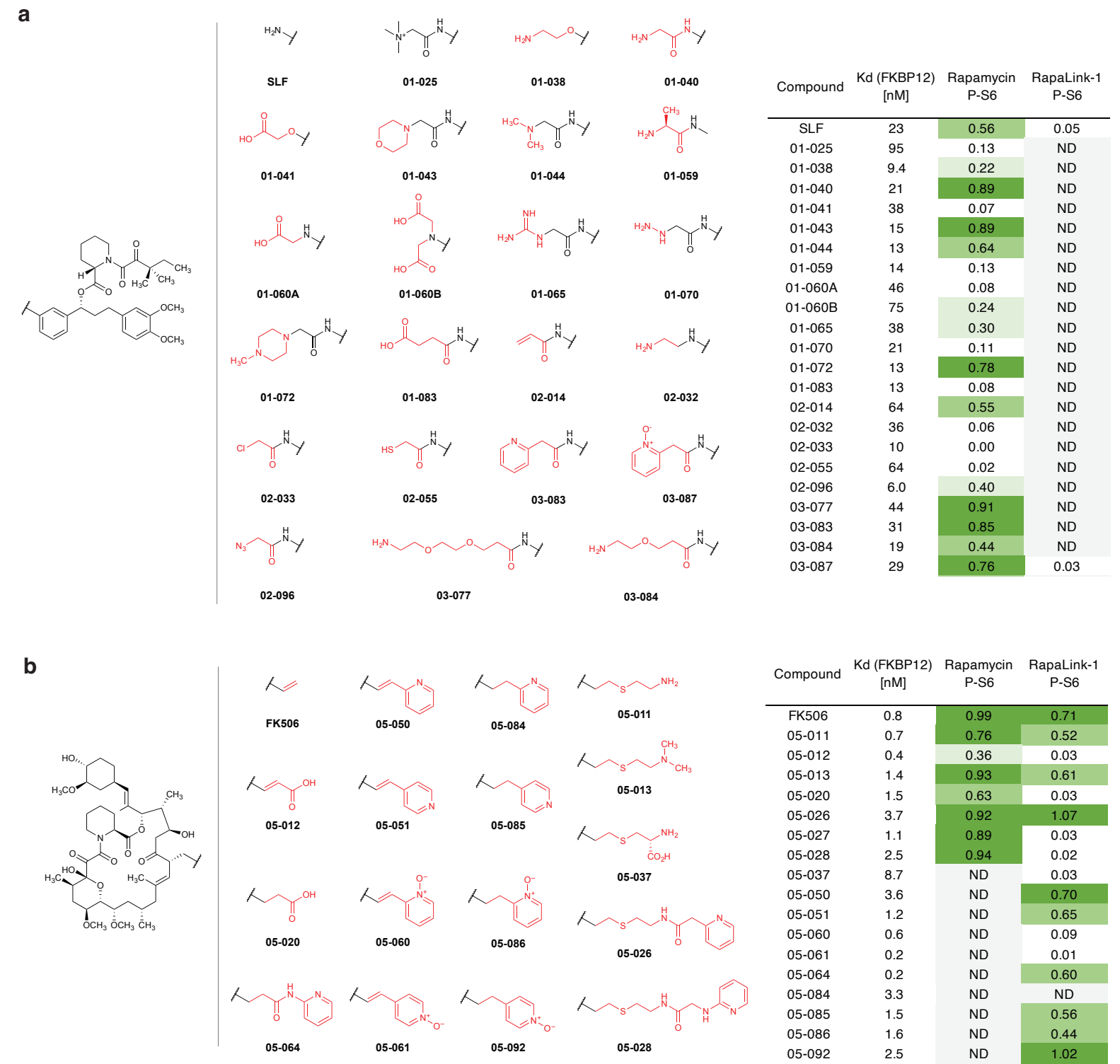

475 Extended Data Figure 2. Structures polar FKBP12 ligands synthesized (a, SLF

476 derivatives; b, FK506 derivatives). Listed in the table are their affinity to recombinant

477 FKBP12 (fluorescence polarization assay) and their efficacy of blocking mTOR inhibition

478 by rapamycin or RapaLink-1 (assessed by western blot analysis of p-S6 level after

479 treatment of MCF7 cells with a combination of $10 \mathrm{nM}$ Rapamycin/Rapalink $+10 \mu \mathrm{M}$

480 candidate compound for 24 h. P-S6 level is quantified as fraction of DMSO control). ND,

481 not determined. 
bioRxiv preprint doi: https://doi.org/10.1101/2020.10.12.336677; this version posted October 12, 2020. The copyright holder for this preprint (which was not certified by peer review) is the author/funder, who has granted bioRxiv a license to display the preprint in perpetuity. It is made available under aCC-BY-NC-ND 4.0 International license.

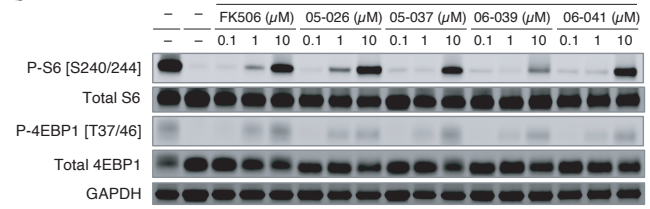

b

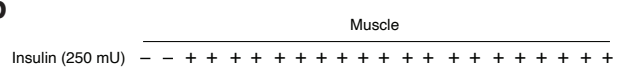$$
\text { Insuln }
$$

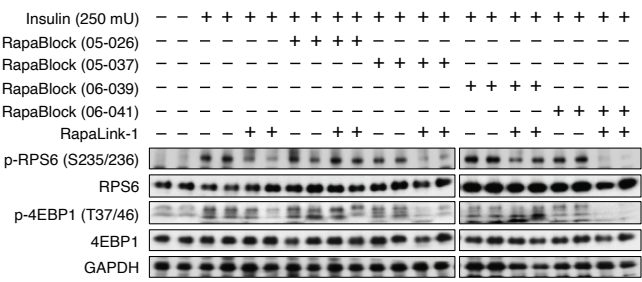

483 Extended Data Figure 3. Four candidate RapaBlock molecules were evaluated in 484 cells (a) and in vivo (b). 
a

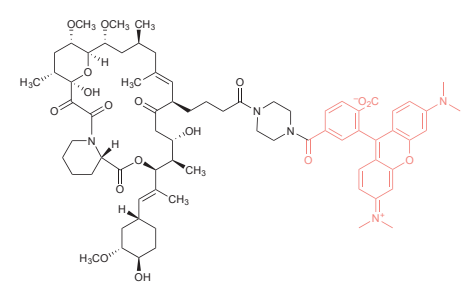

FK-TAMRA b

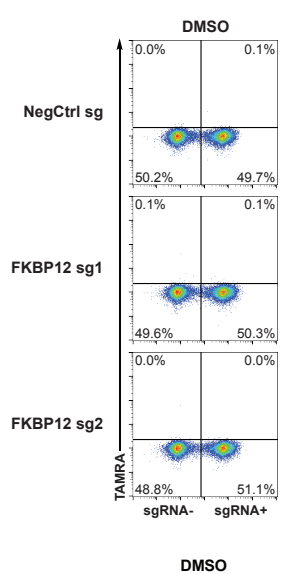

DMso

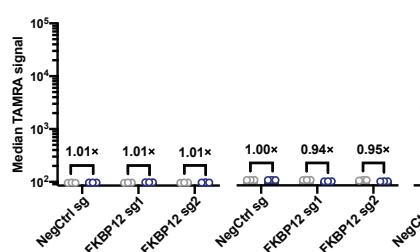

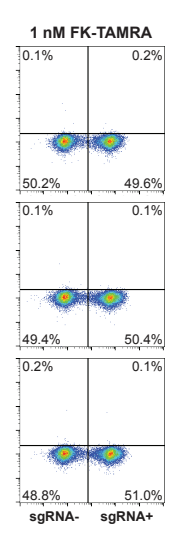

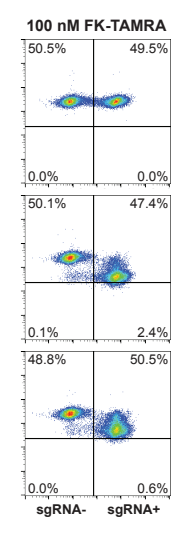

$100 \mathrm{nM}$ FK-TAMRA

487 interaction contributes to cellular accumulation of a fluorescence analog of FK506.

b, FKBP12 knockdown decreases

489 cellular retention of FK-TAMRA. See Supplementary Information for gating strategy. 
a

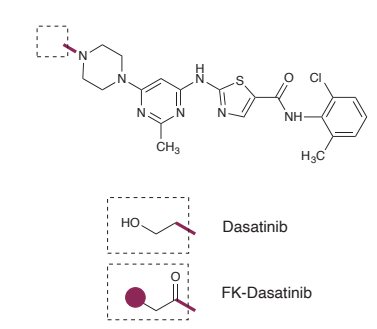

c

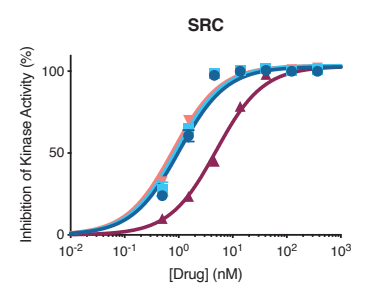

e

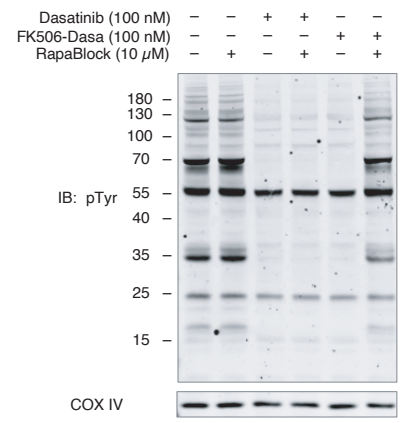

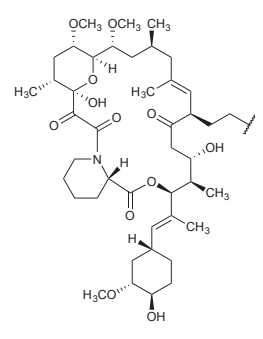

= FK506 C21 Fragment

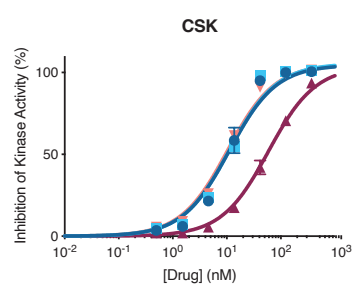

$=$ Dasatinib $+10 \mu \mathrm{M}$ FKBP12 $\quad$ FK-Dasatinib b

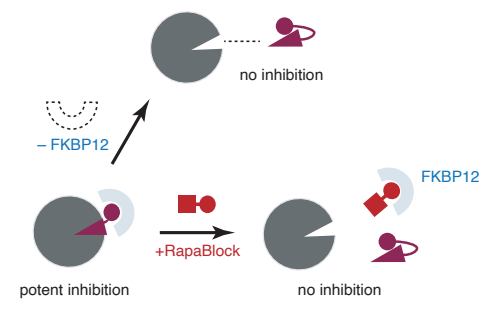

d

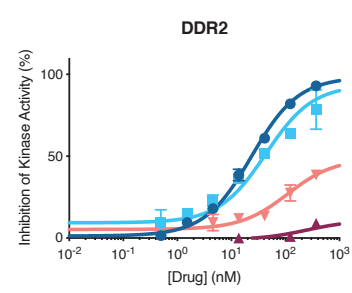

F FK-Dasatinib + $10 \mu \mathrm{M} \mathrm{FKBP12}$

f
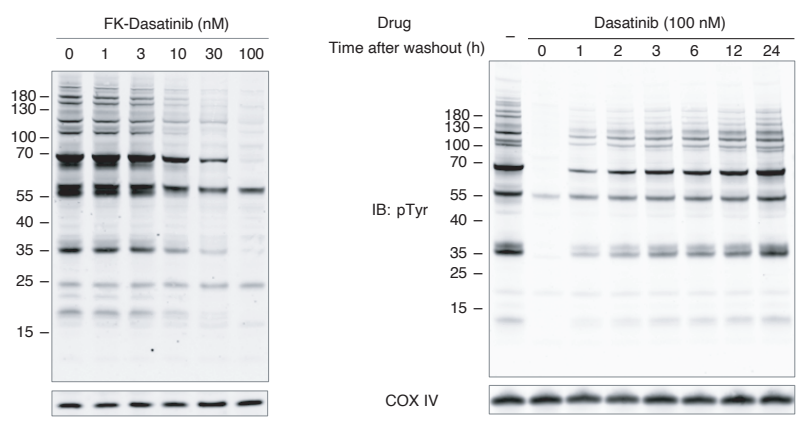

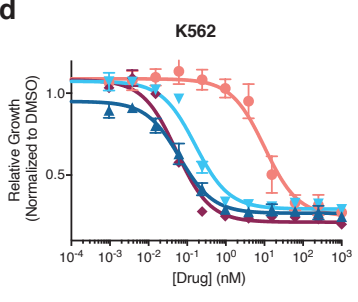

- Dasatinib $\quad$ Dasatinib $+10 \mu \mathrm{M}$ RapaBlock — DK-Dasatinib — FK-Dasatinib + $10 \mu \mathrm{M}$ RapaBlock

g

h
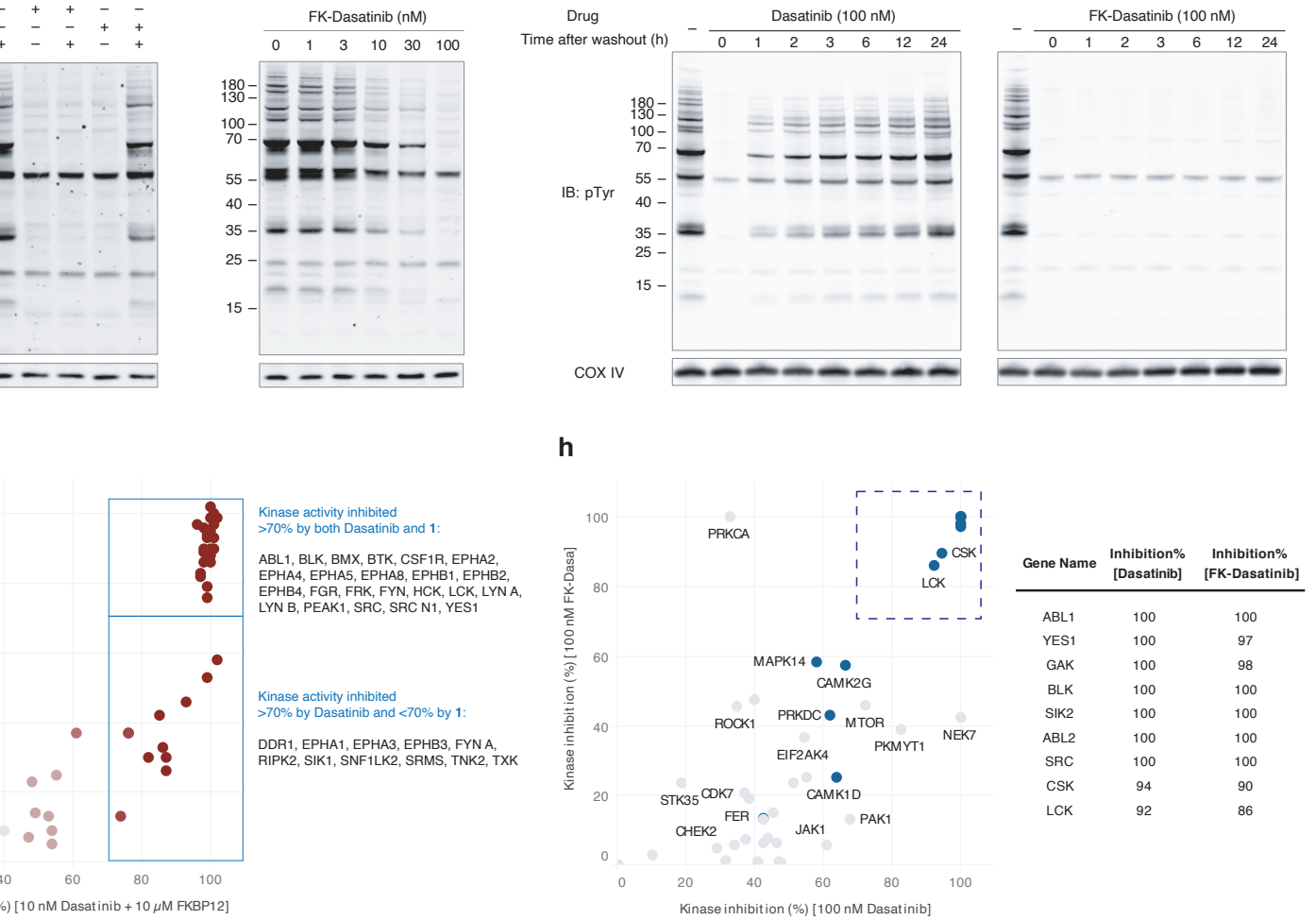

491 Extended Data Figure 5. FK-Dasatinib is a FKBP12-dependent Src family kinase

492 inhibitor with long cellular retention time. a, Structures of Dasatinib and FK-Dasatinib.

493 b, Proposed working model for FKBP-dependent kinase inhibitors. c, Inhibition of Src, 494 Csk and DDR2 kinases by Dasatinib and FK-Dasatinib in the absence or presence of 495 supplemented $10 \mu \mathrm{M}$ recombinant FKBP12 protein. Data is the average of two replicates. 
496 d, Inhibition of K562 cell proliferation by Dasatinib and FK-Dasatinib, in the presence or 497 absence of $10 \mu \mathrm{M}$ RapaBlock. Data is the average of three replicates. e, Jurkat cells were 498 stimulated with anti-CD3 antibody (OKT3) in the presence of dasatinib, FK-dasatinib, 499 and/or RapaBlock and analyzed by immunoblot. f, Jurkat cells were pulse-treated with 500 Dasatinib (100 nM) or FK-Dasatinib (100 nM) for $1 \mathrm{~h}$, then drugs were washed out and 501 cells were incubated in drug-free media for various amounts of time, stimulated with anti502 CD3 antibody (OKT3) for $5 \mathrm{~min}$ and analyzed by immunoblot. $\mathbf{g}$, inhibition of 476 purified 503 kinases by Dasatinib $(10 \mathrm{nM})$ or FK-Dasatinib $(10 \mathrm{nM})$ in the presence of $10 \mu \mathrm{M}$ 504 recombinant FKBP12 protein. $\mathbf{h}$, kinase profiling of Dasatinib (100 nM Dasatinib) or FK505 Dasatinib (100 nM) in Jurkat cells using the covalent occupancy probe XO44. See 506 Supplementary Information for experimental details. 
a

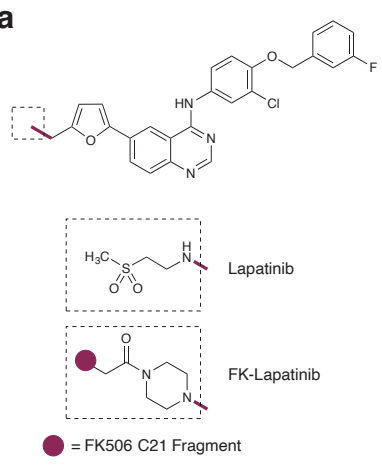

C

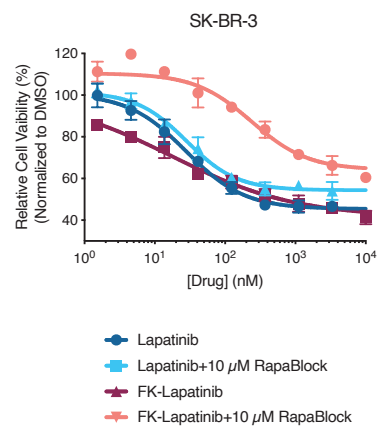

b
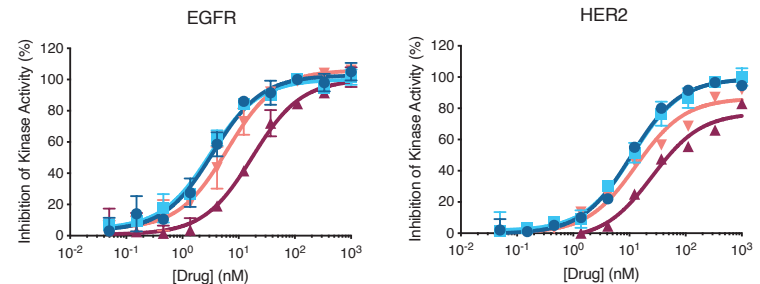

- Lapatinib - Lapatinib+10 $\mu \mathrm{M}$ FKBP12 - FK-Lapatinib - FK-Lapatinib+10 $\mu \mathrm{M}$ FKBP12

d

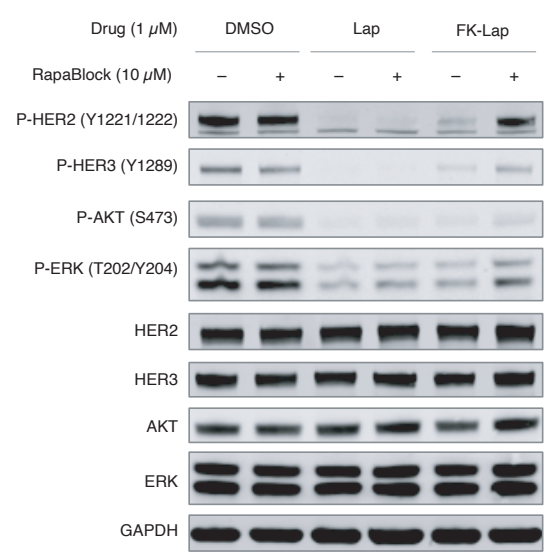

508 Extended Data Figure 6. FK-Lapatinib is a FKBP12-dependent EGFR/HER2 kinase

509 inhibitor. a, Chemical structures of Lapatinib and FK-Lapatinib. b, Inhibition of EGFR

510 and HER2 kinase activity by Lapatinib and FK-Lapatinib in the absence or presence of

511 supplemented $10 \mu \mathrm{M}$ recombinant FKBP12 protein. Data is the average of two replicates.

512 c, Inhibition of proliferation of SK-BR3 cells by lapatinib and FK-lapatinib in the presence

513 of absence of $10 \mu \mathrm{M}$ RapaBlock. Data is the average of three replicates. $\mathbf{d}$, SK-BR3 cells

514 were treated with Lapatinib, FK-Lapatinib, and/or RapaBlock for $1 \mathrm{~h}$ and analyzed by

515 immunoblot. 
a

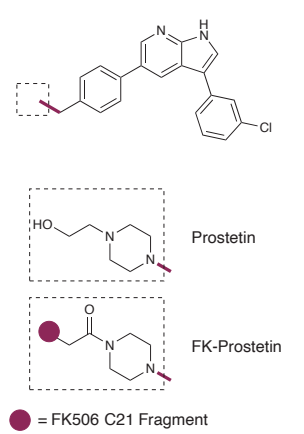

b

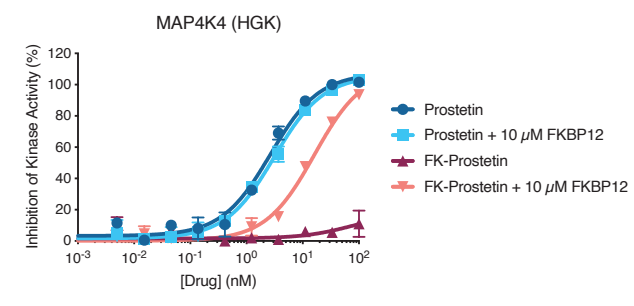

517 Extended Data Figure 7. FK-Prostetin is a FKBP12-dependent MAP4K4(HGK)

518 kinase inhibitor. a, Chemical structures of Prostetin and FK-Prostetin. b, Inhibition of

519 MAP4K4(HGK) kinase activity by Lapatinib and FK-Lapatinib in the absence or presence

520 of supplemented $10 \mu \mathrm{M}$ recombinant FKBP12 protein. Data is the average of two 521 replicates. 


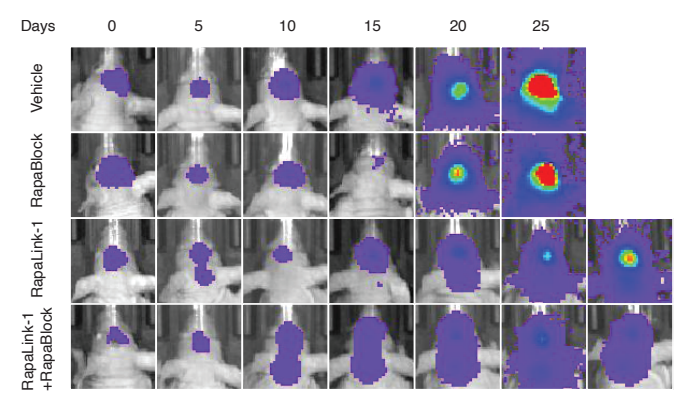

\section{Extended Data Figure 8. Exemplary bioluminescence images of mice bearing}

524 intracranial glioblastoma xenografts. U87MG cells expressing firefly luciferase were

525 injected intracranially into BALB/c ${ }^{\text {nu/nu }}$ mice. After tumor establishment, mice were sorted 526 into four groups and treated by i.p. injections every 5 days of vehicle, RapaBlock ( $1 \mathrm{mg} / \mathrm{kg})$, 527 RapaBlock (40 mg/kg), or a combination of RapaLink-1 and RapaBlock (1 mg/kg and 40 $528 \mathrm{mg} / \mathrm{kg}$, respectively). Bioluminescence imaging of tumor-bearing mice was obtained at 529 days shown (day 0 was start of treatment), using identical imaging conditions. 